\title{
Genome-wide association studies using an adaptive two-stage analysis for a case-control design Kijoung Song*1, Qing Lu ${ }^{2}$, Xiwu Lin ${ }^{1}$, Dawn Waterworth ${ }^{1}$ and Robert C Elston²
}

Address: ${ }^{1}$ GlaxoSmithKline, 709 Swedeland Road, UW 2111, King of Prussia, Pennsylvania 19406, USA and ${ }^{2}$ Department of Epidemiology and Biostatistics, Case Western Reserve University, Case Western Reserve University, 2103 Cornell Road, Wolstein Research Building, Room 1304, Cleveland, Ohio 44106, USA

Email: Kijoung Song* - kijoung.2.song@gsk.com; Qing Lu - qlu@darwin.epbi.cwru.edu; Xiwu Lin - xiwu.2.lin@gsk.com; Dawn Waterworth - dawn.m.waterworth@gsk.com; Robert C Elston - rce@darwin.epbi.cwru.edu

* Corresponding author

from Genetic Analysis Workshop 15

St. Pete Beach, Florida, USA. II-15 November 2006

Published: 18 December 2007

BMC Proceedings 2007, I (Suppl I):SI 47

This article is available from: http://www.biomedcentral.com/I753-656I/I/SI/SI 47

(C) 2007 Song et al; licensee BioMed Central Ltd.

This is an open access article distributed under the terms of the Creative Commons Attribution License (http://creativecommons.org/licenses/by/2.0), which permits unrestricted use, distribution, and reproduction in any medium, provided the original work is properly cited.

\section{Background}

The advance of genotyping technologies and reduction of genotyping costs are resulting in genome-wide association studies using 100,000 to 500,000 (100 k-500 k) singlenucleotide polymorphisms (SNPs) across the whole genome in which tests for association are performed between each SNP and a disease in a case-control design. However, one of the big challenges of genome-wide association studies is the issue of multiple testing [1].
Zhang et al. [2] proposed an adaptive two-stage (ATS) analysis using two trend tests, the Hardy-Weinberg disequilibrium trend test (HWDTT) and the Cochran-Armitage trend test (CATT). All samples are used in both stages in the same way that Van Steen et al. [3] applied a twostage analysis of family-based association. The adaptive two-stage analysis proposed here uses the HWDTT in the first stage to screen the SNPs and then tests a reduced number of SNPs that pass this screening step using the 
CATT in the second stage. The conservative Bonferronicorrected $p$-value of an allele-based test is obtained for each SNP, but it is only necessary to correct for the number of SNPs included in the second-stage analysis.

As an alternative approach, Fisher's combination of $p$-values, referred to as Fisher's test, was considered by Zhang et al. [2]. Because the HWDTT and CATT are asymptotically independent under the null hypothesis, Fisher's test statistic [4] is given by $T=-2 \log \left(p_{\text {HWDTT }}\right)-2 \log \left(p_{\text {CATT }}\right)$, which under the null has a chi-square distribution with $4 \mathrm{df}$. Recently, Hwang et al. [5] extended Fisher's test to a "weighted" version that aims to maximize overall statistical power for a given significance level $(0 \leq \alpha \leq 1)$ using a nonparametric distribution with a Gaussian Kernel density.

In this study, we applied the ATS analysis to the Genetic Analysis Workshop (GAW15) simulated data set in order to find susceptibility disease genes. We compared the results of the ATS with those of the CATT, the HWDTT, the classical Fisher test (CFT), and the weighted Fisher test (WFT). The ATS was Bonferroni-corrected for multiple testing, so, for the sake of comparison, the CATT, HWDTT, and Fisher tests (CFT and WFT) are also adjusted using the same correction method.

\section{Methods}

For the ATS analysis of association, Zhang et al. [2] applied the HWDTT and the CATT to case-control studies. Song and Elston [6] and Zhang et al. [2] showed that these two statistics are asymptotically independent under the null hypothesis of no association. Therefore, they used all samples for both stages of the analysis. For the first stage of the proposed ATS analysis, the HWDTT is applied to test each SNP at the significance level $\alpha_{1}$ chosen on the basis of the conditional power of the HWDTT. The smallest $\alpha_{1}$ is chosen such that the power is at least $1-\beta$, where $\beta$ is the type II error.

Denote the estimators of the genotype frequencies in cases and controls $\hat{p}_{i}=r_{i} / r$ and $\hat{q}_{i}=s_{i} / s$ for $i=0,1,2$, so that $\hat{p}_{A}=\hat{p}_{2}+\hat{p}_{1} / 2$ and $\hat{q}_{A}=\hat{q}_{2}+\hat{q}_{1} / 2$ are estimators of the frequencies of the allele $A$ in cases and controls. Song and Elston [6] considered the difference in disequilibrium coefficients between cases $\left(D_{1}\right)$ and controls $\left(D_{0}\right)$, where $D_{1}=p_{2}-\left(p_{2}+p_{1} / 2\right)^{2}$ and $D_{0}=q_{2}-\left(q_{2}+q_{2} / 2\right)^{2}$. The HWDTT statistic can be written as

$T_{H W D T T}=\frac{Z_{H W D T T}^{2}}{\hat{\operatorname{Var}}\left(Z_{\text {HWDTT }}\right)}=\frac{r s n^{3}\left[\left(\hat{p}_{2}-\hat{p}_{A}^{2}\right)-\left(\hat{q}_{2}-\hat{q}_{A}^{2}\right)\right]^{2}}{\left\{n-\left(n_{2}+n_{1} / 2\right)\right\}^{2}\left(n_{2}+n_{1} / 2\right)^{2}}$, where $n_{i}=\left(r_{i}+s_{i}\right)$ and $n=\sum_{i=0}^{2} n_{i}$. The asymptotic power of the HWDTT can then be written as

$\pi=\Phi\left(\frac{-z_{1-\alpha_{1} / 2} \sigma_{0}-\sqrt{n}\left(D_{1}-D_{0}\right)}{\sigma_{H}}\right)+1-\Phi\left(\frac{z_{1-\alpha_{1} / 2} \sigma_{0}-\sqrt{n}\left(D_{1}-D_{0}\right)}{\sigma_{H}}\right)$,

where

$f(a, b)=(1-2 b-a)^{2} b(1-b)+2 a b(b+a / 2)(1-2 b-a)+(b$ $+a / 2)^{2} a(1-a)$,

$\sigma_{0}^{2}=f\left(r^{\prime} p_{1}+s^{\prime} q_{1}, r^{\prime} p_{2}+s^{\prime} q_{2}\right) /\left(r^{\prime} s^{\prime}\right), r^{\prime} \approx r / n$ and $s^{\prime} \approx s / n$,

$\sigma_{H}^{2}=n \operatorname{Var}\left(D_{1}-D_{0}\right)$,

$\Phi$ is the distribution function of the standard normal $N(0$, $1)$, and

$z_{1-\alpha_{1} / 2}$ is the $100\left(1-\alpha_{1} / 2\right)^{\text {th }}$ percentile of $N(0,1)$.

The SNPs for which the null hypotheses are rejected in the first stage are tested in the second stage analysis by the CATT at the level $\alpha_{2}=\alpha^{\prime} /\left(m \alpha_{1}\right)$, where $\alpha^{\prime}$ is obtained by the parametric bootstrap to control the overall type I error rate of the ATS analysis. Then $\alpha_{2}$ controls the overall type I error rate to $\alpha$ (taken to be 0.05 ) for a total of $m$ simultaneous hypothesis tests (SNPs). As in Van Steen et al. [3], the overall $p$-value of the ATS is the $p$-value of the second analysis, which here is the CATT.

\section{Data}

We used the GAW15 simulated Problem 3 data set for rheumatoid arthritis (RA), which includes 100 replicates. Each replicate contains 1500 families with an affected sibling pair and 2000 unaffected control subjects. To obtain a sample of cases and controls, we randomly chose one case from each affected sib pair. Thus, from each replicate we selected 1500 cases with RA and 2000 controls. In order to compare the performance of all methods in a small sample size, we randomly sampled 200 cases and 200 controls from each of the 100 replicate samples of 1500 cases and 2000 controls. To examine type I error rate, we concentrated on 100 markers that were at least $20,000 \mathrm{~kb}$ distant from the identified peaks on chromosome 6 . Therefore, the total number of marker tests to examine type I error was 10,000 (100 markers $\times 100$ replicates). To examine power, we concentrated on all 674 markers on chromosome 6 using only one replicate of 200 cases and 200 controls. Among these 674 markers, 5 markers are causative, in the region between 32447.149 $\mathrm{kb}$ and $37363.880 \mathrm{~kb}$ on chromosome 6 . 


\section{Results}

The results for type I error are shown in Table 1 for the nominal significance levels $\alpha=0.05$ and $\alpha=0.001$. Table 1 shows that all the test statistics have nominal significance level close to the actual significance levels.

Without multiple testing corrections, the HWDTT, CATT, CFT, and WFT showed strong associations $(p<0.001)$ with a susceptibility disease gene in the region (Fig. 1, green line) between $32447.149 \mathrm{~kb}$ and $32859.819 \mathrm{~kb}$ on chromosome 6 . In addition, the HWDTT, CATT, and Fisher tests (CFT and WFT) showed a significant association $(p<0.05)$ at $37363.880 \mathrm{~kb}$, which was close to a trait locus $(37233.784 \mathrm{~kb})$. The results also indicated that i) CATT and CFT have similar power near the peak, ii) CFT is more powerful than WFT near the peak, and iii) the HWDTT is not powerful near the peak.

With Bonferroni multiple testing correction, Table 2 shows the locations of the SNP markers that are significantly associated with RA. In Table 2, the p-values for HWDTT, CATT, WFT, and CFT are compared at the significance level $0.05 / 674=7.42 \times 10^{-5}$. To obtain the $p$-value of the ATS analysis, first we calculated the asymptotic power of the HWDTT for $\alpha_{1}=0.01$ to 1.00 with increments of 0.01 for each of the 674 SNPs. Then for each SNP we chose the $\alpha_{1}$ that had at least $80 \%$ conditional power. Results showed that 535 SNPs had p-values of HWDTT less than this $\alpha_{1}$ and these were analyzed in the second stage. Then we obtained the adjusted overall level for $\alpha^{\prime}$ using the parametric bootstrap with increments of 0.001 and 10,000 replications. For example, for $80 \%$ conditional power, the SNP in location $32447.149 \mathrm{~kb}$ requires $\alpha_{1}=0.77$ in the first stage. Then, based on the bootstrap, we obtained $\alpha^{\prime}=0.03$. Thus, the adjusted level required for the second stage is $\alpha_{2}=0.03 /(674 \times 0.77)=5.78 \times 10^{-}$ ${ }^{5}$. The $p$-values of the HWDTT is $0.53<\alpha_{1}=0.77$. Thus, the $\mathrm{SNP}$ in location $32447.149 \mathrm{~kb}$ is significant in the first stage. The $p$-value of the CATT in the second stage is $2.7 \times$ $10^{-15}<\alpha_{2}=5.8 \times 10^{-5}$. Hence, the SNP in location $32447.149 \mathrm{~kb}$ is significant when the adaptive two-stage analysis is applied.

Applying the ATS with the optimal $\alpha_{2}$, the three SNPs in the region between $32447.149 \mathrm{~kb}$ and $32772.203 \mathrm{~kb}$ were associated with RA. In particular, after Bonferroni correction the ATS showed that the SNP at $37363.880 \mathrm{~kb}$ was associated with RA. In the region between $32447.149 \mathrm{~kb}$ and $32859.819 \mathrm{~kb}$, the CATT and CFT had Bonferroni-corrected average $p$-values $<1.34 \times 10^{-12}$ and $p<5.80 \times 10^{-7}$, respectively. In addition, the corrected $p$-value of CFT is 0.000802 for a marker at location $37363.880 \mathrm{~kb}$. However, the Bonferroni-corrected $p$-value of WFT is not significant. This could indicate that WFT with Bonferroni correction may be too conservative, because it already minimizes the number of false positives and false negatives.

\section{Discussion}

We have presented the use of a new method for genomewide association studies with optimal choice of significance level to maximize the power and at the same time asymptotically control the overall type I error. The ATS analysis uses two independent test statistics-here the HWDTT and the CATT. We compared the performance of the HWDTT, CATT, Fisher's tests combining the HWDTT and CATT, and the ATS in this data set with Bonferroni correction for multiple testing and found that the ATS had good performance. Compared to WFT or CFT, the ATS showed higher power in this study. CFT captured the region that is close to a trait locus and gave higher power than WFT, but had $p<0.05$ in regions that were distant from the true locations, so that the falsepositive rate of CFT seems to be higher than that of WFT in these data. This agrees with the original paper [5] reporting a lower false-positive rate for WFT.

In this study, we applied all test statistics to the GAW15 simulated data. However, the simulated effect near the peak is so strong that all test statistics were able to detect the susceptibility disease gene on chromosome 6 with a sample size as small as 200 cases and 200 controls; the Bonferroni corrected $p$-values were significant for the CATT, CFT, and ATS. Zhang et al. [2] showed that the ATS is more powerful than the CATT and CFT when applied to real data in an association study of 96 cases and 50 controls that used 103,611 SNPs for a genome-wide association study of age-related macular degeneration [7].

Table I: Type I error rates

\begin{tabular}{llc}
\hline Test statistics & $\alpha=0.05$ & $\alpha=0.001$ \\
\hline Hardy-Weinberg disequilibrium trend test & 0.0468 & 0.0013 \\
Cochran-Armitage trend test & 0.0488 & 0.0008 \\
Classical Fisher's test & 0.0503 & 0.0017 \\
Weighted Fisher's test & 0.0502 & 0.0010 \\
Adaptive two-stage & 0.0492 & 0.0012
\end{tabular}



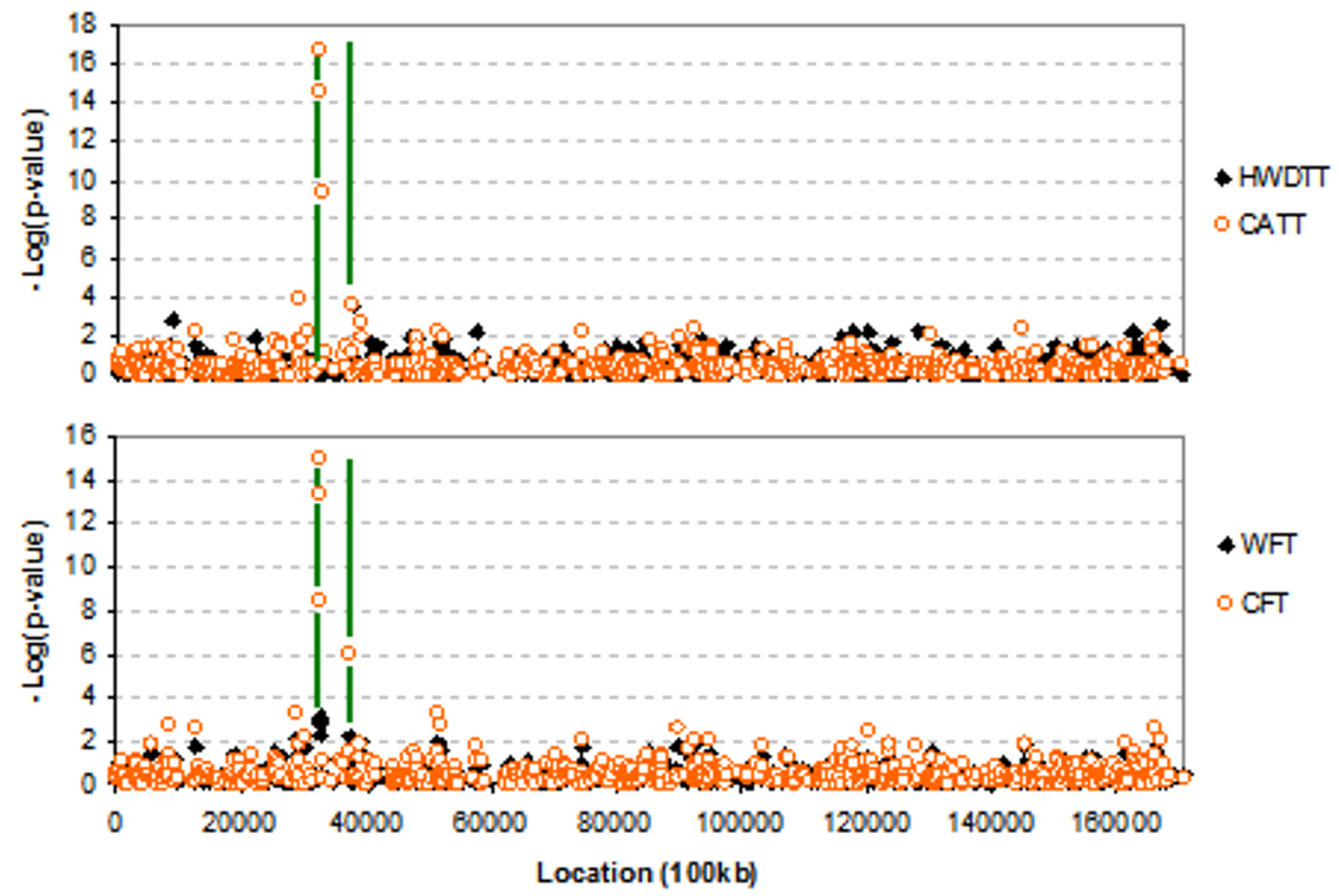

Figure I

Results of SNP markers using HWDTT, CATT, CFT, and WFT for chromosome 6.

Table 2: The locations of the SNP markers that are significantly associated with RA after Bonferroni correction at the significance level $\alpha=0.05$

\begin{tabular}{|c|c|c|c|c|c|c|}
\hline \multirow[b]{2}{*}{ Location (kb) } & \multirow[b]{2}{*}{ HWDTTa } & \multirow[b]{2}{*}{ CATTa } & \multirow[b]{2}{*}{ WFTa } & \multirow[b]{2}{*}{$\mathrm{CFTa}^{\mathrm{a}}$} & \multicolumn{2}{|c|}{ ATS } \\
\hline & & & & & Stage Ic & Stage $2^{d}$ \\
\hline 32447.149 & NSe & $2.65 \times 10^{-15}$ & NS & $4.96 \times 10^{-14}$ & $0.53<0.77$ & $2.7 \times 10^{-15}<5.8 \times 10^{-5}$ \\
\hline 32499.465 & NS & $<1.0 \times 10^{-17}$ & NS & $<1.10 \times 10^{-16}$ & $0.65<0.83$ & $1.0 \times 10^{-17}<6.3 \times 10^{-15}$ \\
\hline 32521.277 & NS & $<1.0 \times 10^{-17}$ & NS & $<1.10 \times 10^{-16}$ & NS & NS \\
\hline 32772.203 & NS & $5.36 \times 10^{-15}$ & NS & $3.44 \times 10^{-9}$ & $0.27<0.65$ & $5.4 \times 10^{-15}<5.7 \times 10^{-5}$ \\
\hline 37363.880 & NS & NS & NS & $1.19 \times 10^{-6}$ & $0.0003<0.02$ & $0.0003<0.001 \mathrm{I}$ \\
\hline
\end{tabular}

aThe $p$-values should be compared with $0.05 / 674=7.42 \times 10^{-5}$.

bThe $p$-value should be compared with the optimal level in the two stages.

cThe level in the first stage with conditional power at least $80 \%\left(\alpha_{1}\right)$

dThe adjusted level for the second stage $\left(\alpha_{2}=\alpha^{\prime} /\left(674 \alpha_{1}\right)\right)$

eNS: no significance 
Finally, we should note the limitations of the ATS method we used. The ATS analysis will be more costly than other two-stage analysis studies, which uses separate portions of the sample for each stage, because the ATS analysis uses all subjects in both stages. In addition, the ATS analysis is more computationally intensive than the other tests because it is a necessary to obtain the adjusted overall level for $\alpha^{\prime}$ using the parametric bootstrap.

\section{Conclusion}

Using the ATS analysis, a sample size as small as 200 cases and 200 controls showed good performance after Bonferroni correction for association with a susceptibility disease gene in the region between $32447.149 \mathrm{~kb}$ and $37363.880 \mathrm{~kb}$ on chromosome 6 .

\section{Competing interests}

The author(s) declare that they have no competing interests.

\section{Acknowledgements}

This article has been published as part of BMC Proceedings Volume I Supplement I, 2007: Genetic Analysis Workshop 15: Gene Expression Analysis and Approaches to Detecting Multiple Functional Loci. The full contents of the supplement are available online at http://www.biomedcentral.com/ $\underline{|753-6561 /| \text { ? issue=SI. }}$.

\section{References}

I. Wang WYS, Barratt BJ, Clayton DG, Todd JA: Genome-wide association studies: theoretical and practical concerns. Nat Rev Genet 2005, 6:109-II8.

2. Zheng G, Song K, Elston RC: Adaptive two-stage analysis of genetic association in case-control designs. Hum Hered 2007, 63:175-186.

3. Van Steen K, McQueen MB, Herbert A, Raby B, Lyon H, DeMeo DL, Murphy A, Su J, Datta S, Rosenow C, Christman M, Silverman EK, Laird NM, Weiss ST, Lange C: Genomic screening and replication using the same data set in family-based association testing. Nat Genet 2005, 37:683-69l.

4. Elston RC: On Fisher's method of combining $p$-values. Biometrical J 1991, 33:339-345.

5. Hwang D, Rust A, Ramsey S, Smith S, Leslie DM, Weston AD, Atauri PD, Aitchison JD, Hood L, Siegel AF, Bolouri H: A data integration methodology for systems biology. Proc Natl Acad Sci USA 2005, I02:|7296-I730I.

6. Song K, Elston RC: A powerful method of combining measures of association and Hardy-Weinberg equilibrium for finemapping in case-control studies. Stat Med 2005, 25: I05-I 26.

7. Klein RJ, Zeiss C, Chew EY, Tsai JY, Sackler RS, Haynes C, Henning AK, SanGiovanni JP, Mane SM, Mayne ST, Bracken MB, Ferris FL, Ott J, Barnstable $\mathrm{C}$, Hoh J: Complement factor $\mathbf{H}$ polymorphism in age-related macular degeneration. Science 2005, 308:385-389.
Publish with Bio Med Central and every scientist can read your work free of charge

"BioMed Central will be the most significant development for disseminating the results of biomedical research in our lifetime. " Sir Paul Nurse, Cancer Research UK

Your research papers will be:

- available free of charge to the entire biomedical community

- peer reviewed and published immediately upon acceptance

- cited in PubMed and archived on PubMed Central

- yours - you keep the copyright

Submit your manuscript here:

http://www.biomedcentral.com/info/publishing_adv.asp
BioMedcentral 